\title{
Has_a Relationship
}

National Cancer Institute

\section{Source}

National Cancer Institute. has a Relationship. NCI Thesaurus. Code C82846.

A relationship of specialization that states that a concept is a superset of another concept. The inverse of the isa relationship. 